Check for updates

Cite this: Mater. Chem. Front., 2020, 4, 2047

Received 17th April 2020 Accepted 25th May 2020

DOI: $10.1039 / \mathrm{d} 0 \mathrm{qm} 00247 \mathrm{j}$

rsc.li/frontiers-materials

\title{
AIE-active multicolor tunable luminogens: simultaneous mechanochromism and acidochromism with high contrast beyond $100 \mathrm{~nm} \dagger$
}

\author{
Wei Yang, ${ }^{\text {ab }}$ Yiyu Yang, ${ }^{\mathrm{b}}$ Yuntao Qiu, ${ }^{\mathrm{b}}$ Xiaosong Cao, (D) ${ }^{\mathrm{b}}$ Zhongyan Huang, $^{\mathrm{b}}$ \\ Shaolong Gong (D) *a and Chuluo Yang (D)*ab
}

\begin{abstract}
The development of intelligent luminescent materials, particularly those exhibiting high-contrast $\left(\Delta \lambda_{\mathrm{em}, \max }>100 \mathrm{~nm}\right)$ and multicolor mechanochromic luminescent $(\mathrm{MCL})$ characteristics, is highly desirable but a formidable challenge. In this work, we designed three luminogens with aggregationinduced emission (AIE) features, namely 7PTP-BPXZ, 5PTP-BPXZ and 5,7PTP-BPXZ. The synergistic effect between the folded conformation of the benzo[a]phenoxazine fragment and various intermolecular non-covalent interactions is beneficial for multicolor tunable emission upon external stimuli. Impressively, 7PTP-BPXZ and 5PTP-BPXZ displayed reversible tricolor MCL behavior with high contrast $\left(\Delta \lambda_{\text {em,max }} 114 \mathrm{~nm}\right.$ and $137 \mathrm{~nm}$, respectively). Furthermore, 7PTP-BPXZ possessed acidochromic function with $\Delta \lambda_{\text {em,max }}$ of $132 \mathrm{~nm}$. This is the first report of single-component small organic molecules featuring both multicolor and high-contrast beyond $100 \mathrm{~nm}$ mechanochromism and acidochromism.
\end{abstract}

\section{Introduction}

Organic solid-state intelligent luminescent materials (or luminogens) are a category of versatile materials with tunable appearance color or emission in response to external stimuli. During the past decade, smart luminogens have drawn intensive research enthusiasm in the fields of mechanosensors, organic light-emitting diodes, organic lasers, security systems, optical storage, etc. ${ }^{1}$ According to stimulating types, the color switching phenomena can be further divided into photochromism, electrochromism, thermochromism, mechanochromism, acidochromism, and so forth. ${ }^{2}$ Specifically, great progress has been made in mechanochromic luminescent (MCL) materials with emission features governed by physical channels, such as anisotropic grinding, shearing or rubbing. These approaches only change molecular stacking patterns or conformations, but do not alter the native chemical structures. Nowadays, plenty of MCL materials involving metal complexes, polymers and pure organic

\footnotetext{
${ }^{a}$ Renmin Hospital of Wuhan University, Department of Chemistry, Hubei Key Lab on Organic and Polymeric Optoelectronic Materials, Wuhan University, Wuhan, 430072, People's Republic of China.E-mail: clyang@whu.edu.cn, slgong@whu.edu.cn

${ }^{b}$ College of Materials Science and Engineering,

Shenzhen University, Shenzhen 518060, People's Republic of China

$\dagger$ Electronic supplementary information (ESI) available: Synthesis and additional figure details. CCDC 1994907-1994909. For ESI and crystallographic data in CIF or other electronic format see DOI: $10.1039 / \mathrm{d} 0 \mathrm{qm} 00247 \mathrm{j}$
}

(metal-free) molecules have been reported to exhibit bicolor switching or low-contrast luminescence, but they are still unsatisfactory for practical applications in anti-counterfeiting complexity and density of optical storage. ${ }^{3}$ Meanwhile, singlecomponent metal-free molecules simultaneously featuring multicolor (more than two colors) and high-contrast mechanochromism (especially with $\Delta \lambda_{\mathrm{em}, \max }>100 \mathrm{~nm}$ ) are underexplored owing to the lack of an effective design strategy.

Aggregation-induced emission (AIE) materials, referring to a type of luminogen which emits faintly in pure solution but shows remarkably enhanced emission in aggregated states, enjoy intrinsic advantages such as multicolor and high-contrast mechanochromic candidates. ${ }^{4}$ These luminogens usually adopt twisted configurations which are facilely manipulated by moderate anisotropic grinding. ${ }^{5}$ Besides, heterocycles, namely cyclic hydrocarbons embedded with heteroatoms (N, O, S, etc.), are often established as building blocks in intelligent luminescent materials. The heteroatoms enable variable conformations of molecules and easily produce intermolecular and intramolecular hydrogen bonds, such as $\mathrm{C}-\mathrm{H} \cdots \mathrm{N}$ and $\mathrm{C}-\mathrm{H} \cdots \mathrm{O}$ bonds, which can result in the modulation of the solid-state emission under the external stimuli. Moreover, luminogens constructed by the combination of large conjugation cores and peripheral phenyl rings may exhibit high-contrast MCL phenomena. Recently, Tang and Li designed an AIE tetraphenylethylene derivative with tricolor mechanochromic properties. It showed fluorescence responses to acid/base vapor, 
and the emission could be altered among blue (462 $\mathrm{nm}$ ), bright cyan (482 $\mathrm{nm}$ ) and yellow (496 nm) by grinding, heating or solvent fuming. ${ }^{6}$ Takeda and Minakata synthesized phenothiazinedibenzophenazine-phenothiazine molecules with tricolor MCL features. ${ }^{7}$ However, the development of simultaneous multicolor and high-contrast mechanochromic small molecules remains a challenging task.

To achieve such a perfect MCL system, the benzo[ $a]$ phenoxazine (BPXZ) unit was chosen as the donor because phenoxazine could adopt the folded conformation and the introduction of the peripheral phenyl ring could further rigidify the molecular architecture. Furthermore, phenyl substituted triazolopyrimidine (PTP) was selected as the acceptor core in virtue of multiple nitrogen atoms which could benefit the formation of weak intermolecular interactions and impede the intramolecular motions in the solid state. Subsequently, three heterocyclic molecules, 12-(4-(7-phenyl-[1,2,4]triazolo[1,5-a]pyrimidin-5-yl)phenyl)-12H-benzo[a]phenoxazine (7PTP-BPXZ), 12-(4-(5-phenyl-[1, $2,4]$ triazolo[1,5-a]pyrimidin-7-yl)phenyl)-12H-benzo[a]phenoxazine (5PTP-BPXZ) and 12,12'-([1,2,4]triazolo[1,5- $a]$ pyrimidine-5,7-diylbis(4,1-phenylene)bis-(12H-benzo[a]phenoxazine)) (5,7PTP-BPXZ) were designed and prepared in this work (Scheme 1). Three luminogens exhibited obvious AIE behaviors which were attributed to the restriction of intramolecular motion in solid states by deeply analyzing their crystal packing arrangements. Significantly, 7PTP-BPXZ and 5PTP-BPXZ showed high-contrast and tricolor MCL features. More impressively, 7PTP-BPXZ displayed a highcontrast $\left(\Delta \lambda_{\mathrm{em}, \max }=132 \mathrm{~nm}\right)$ acidochromic characteristic as well. To the best of our knowledge, this is the first example of single-component small organic molecules with mechanochromism and acidochromism manifesting both multi colors and high contrast $\left(\Delta \lambda_{\mathrm{em}, \max }>100 \mathrm{~nm}\right)$.

\section{Results and discussion}

\section{Synthesis and characterization}

The synthetic route of the compounds is outlined in Scheme S1 (ESI $\dagger$ ). The donor segment (BPXZ) was synthesized in a threestep procedure involving aromatic nucleophilic substitution, nitro-reduction by Fe powder and palladium-catalyzed Buchwald-Hartwig coupling reaction. The PTP acceptors were synthesized according to the previous protocols. ${ }^{8}$ Finally, the target compounds were assembled via an intermolecular

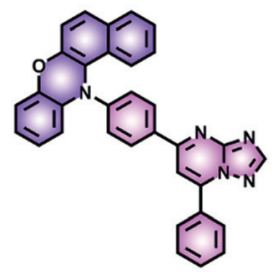

7PTP-BPXZ
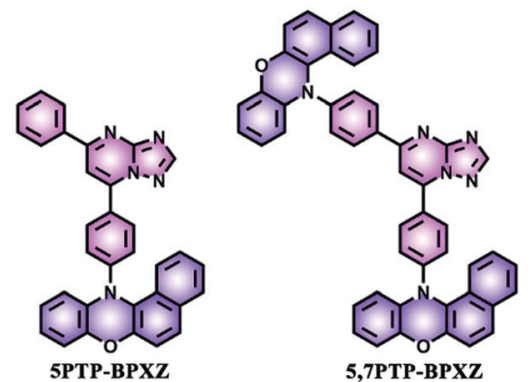

5,7PTP-BPXZ

Scheme 1 Chemical structures of the luminogens.

Buchwald-Hartwig amination reaction between BPXZ and PTP acceptors. The final products were carefully characterized by ${ }^{1} \mathrm{H}$ NMR, ${ }^{13} \mathrm{C}$ NMR, high-resolution mass spectrometry and single-crystal X-ray diffraction.

\section{Theoretical simulation and electrochemical properties}

Density functional theory (DFT) calculations were implemented to predict the frontier molecular orbitals (FMOs) and electronic states of the molecules. As shown in Fig. 1, all BPXZ subunits were largely folded along their central $\mathrm{N} \cdots \mathrm{O}$ axis and adopted quasi-axial (QA) conformations with small donor-acceptor torsion angles $\left(\theta_{\mathrm{a}}\right)$ of $9-11^{\circ}$. The QA geometries facilitated the conjugation between nitrogen lone pair electrons with the adjacent acceptor moiety, resulting in large overlap of the highest occupied molecular orbitals (HOMOs) and lowest unoccupied molecular orbitals (LUMOs) on the PTP units and more localized electronic transitions upon excitation.

The luminogens displayed a reversible one-electron oxidation process in the cyclic voltammetry (CV) curves (Fig. S1, $\mathrm{ESI} \dagger$ ). According to the onset voltages of the oxidation curves, the HOMO levels of 7PTP-BPXZ, 5PTP-BPXZ and 5,7PTP-BPXZ were determined to be $-5.06 \mathrm{eV},-5.13 \mathrm{eV}$ and $-5.10 \mathrm{eV}$, respectively. The LUMO levels deduced from the HOMOs and corresponding energy gaps $\left(E_{\mathrm{g}} \mathrm{s}\right.$, obtained from their absorption onset) were individually calculated to be $-2.13 \mathrm{eV},-2.19 \mathrm{eV}$ and $-2.17 \mathrm{eV}$. These results matched well with the theoretical analysis.

\section{Basic photophysical properties}

The UV-vis absorption and fluorescence spectra of 7PTP-BPXZ, 5PTP-BPXZ and 5,7PTP-BPXZ were measured in solvents with different polarity (i.e. $n$-hexane, toluene, tetrahydrofuran (THF),

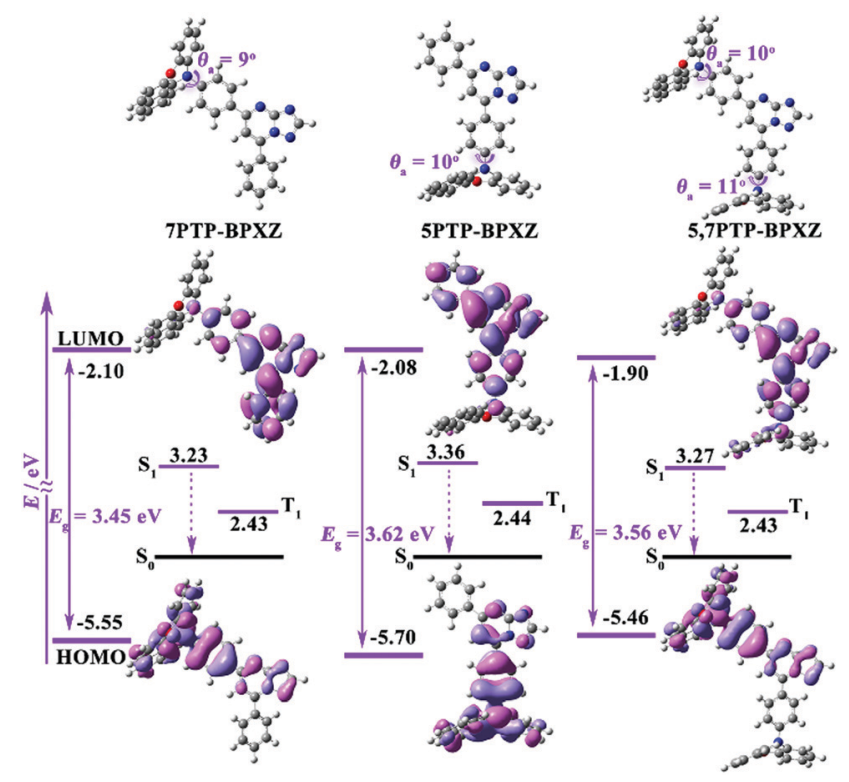

Fig. 1 The optimized configurations and HOMO/LUMO distributions of the luminogens evaluated using the B3LYP-D3(BJ)/def2-SVP level, and the energy level diagram for $S_{1}$ and $T_{1}$ states calculated with TD-DFT:PBEO/ def2-SVP. 
chloroform), and the pertinent photophysical data are summarized in Table S1 (ESI $\dagger$ ). As shown in Fig. S2 (ESI $\dagger$ ), three luminogens exhibited similar strong absorption peaks at around $350 \mathrm{~nm}$ assigned to the local $\pi-\pi^{*}$ transition. However, no obvious absorption from the charge transfer state was observed, indicating favorable QA conformation in the solution state. The fluorescence spectra of all luminogens displayed unstructured emissions. The emission bands underwent a bathochromic shift with decreased intensity when the solvent polarity was increased, mainly ascribed to the intense positive solvation effect and the increased detrimental non-radiative decay in polar media.

To investigate their AIE features, THF/water mixtures with different water fractions $\left(f_{\mathrm{w}} \mathrm{s}\right)$ were utilized as a solvent system. As depicted in Fig. 2, three luminogens exhibited typical AIE properties. Taking 5PTP-BPXZ as an example, the emission was extremely weak with $f_{\mathrm{w}}$ below $80 \%$. Afterwards, the emission intensity was boosted markedly along with further raising $f_{\mathrm{w}}$ owing to the restriction of intramolecular rotations (RIRs) by molecular aggregation, and reached the maximum at $f_{\mathrm{w}}$ of $99 \%$, which was 348-fold higher than that in pure THF solution. Additionally, the aggregates excluded the numerous solvent molecules with high polarity, and thus the luminogen felt less relaxed and the intramolecular charge transfer process became weakened. Consequently, the emission was strengthened and hypochromically shifted in their aggregation states. In the case of 7PTP-BPXZ and 5,7PTP-BPXZ, their maximum emission intensity in the aggregated state was 160 -fold and 168-fold
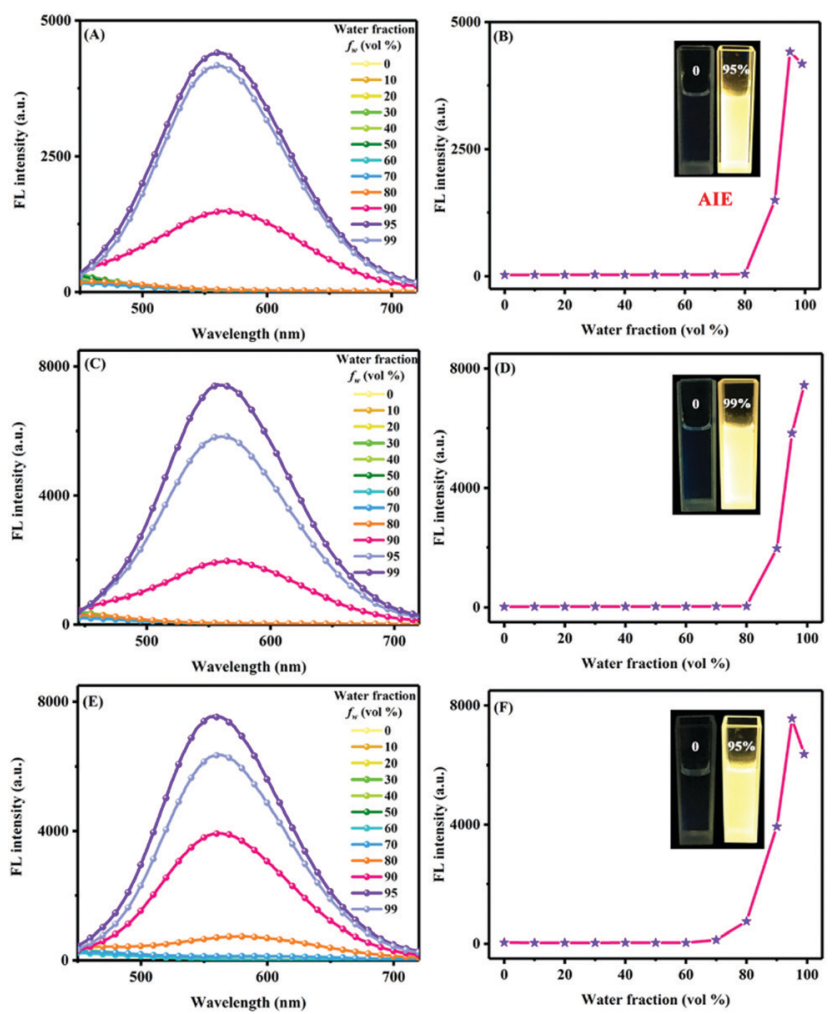

Fig. 2 The fluorescence spectra of luminogens and plot of fluorescence intensity in aqueous mixtures. A/B: 7PTP-BPXZ; C/D: 5PTP-BPXZ; E/F: 5,7PTP-BPXZ. Inset: Photographs under UV illumination. higher than that in THF, respectively. Unlike 5PTP-BPXZ, their emission was slightly decreased when $f_{\mathrm{w}}$ was $99 \%$. There was a possible reason that the molecules might cluster together to form ordered aggregates when $f_{\mathrm{w}}$ was $80-95 \%$, which contributed to the emission enhancement. ${ }^{9,10 a}$ Nevertheless, the molecules might commence agglomerating to form amorphous aggregates while $f_{\mathrm{w}}$ was $99 \%$, which was detrimental to the emission.

In order to gain further insights into the geometrical conformations and packing modes, crystals of 7PTP-BPXZ, 5PTPBPXZ and 5,7PTP-BPXZ were cultivated by solvent diffusion of hexane into the chloroform solution at room temperature. As shown in Fig. S3 (ESI $\dagger$ ), three crystals adopted QA conformations with comparable bending angles $\left(\theta_{\mathrm{b}}\right)$. The dihedral angles $\left(\theta_{\mathrm{a}}\right)$ between donors and acceptors were small, in good accordance with the theoretical predictions. It should be noted that the folded conformation of benzo[ $a]$ phenoxazine substitution is facilely manipulated by external stimuli, which may contribute to their multicolor tunable emission. Crystal 7PTP-BPXZ was a monoclinic system with the space group $P 21 / n$. It could be seen that the dimer with two kinds of intermolecular interactions (Fig. 3A), such as C15-H15 $\cdots \mathrm{N} 4(d=2.90 \AA)$ and $\mathrm{C} 21-\mathrm{H} 21 \cdots \pi$ (namely ring1, C23-C24-C25-N3-C26-N2, $d=3.42 \AA$ ), was involved in the unit cell of 7PTP-BPXZ. Then, these dimers formed a one-dimensional (1D) chain through C30-H30 $\cdots \pi$ (ring2, C11-C12-C13-C14-C15-C16, $d=3.22 ~ \AA$ ) and C30-H30 $\cdots \pi$ (ring3, C28-C29-C30-C31-C32-C33, $d=3.49 \AA$ ) interactions (Fig. 3B). Furthermore, 2D layers were constructed through C3-H3 $\cdots \pi$ (ring4, C1-C2-C3-C4-C5-C6, $d=3.19 \AA$ A), C3-H3 $\cdots \pi$ (ring5, C5-C6-C7-C8-C9-C10, $d=2.88 \AA$ ), C4-H4 $\cdots \pi$ (ring4, $d=3.14 \AA$ ) and $\mathrm{C} 10-\mathrm{H} 10 \cdots \pi$ (ring2, $d=2.83 \AA$ ) (Fig. 3C and D), which suppressed the intramolecular motion and blocked the nonradiative decay channel to emit intense light in the aggregated state. Besides, these weak intermolecular non-covalent interactions are easily varied upon external stimuli, which are also beneficial for the switchable emission.

Crystal 5PTP-BPXZ was a monoclinic system with the space group $P 21$. As depicted in Fig. 4, $\mathrm{CHCl}_{3}$ molecules were entrapped in the crystalline arrangement and held the dimers together.
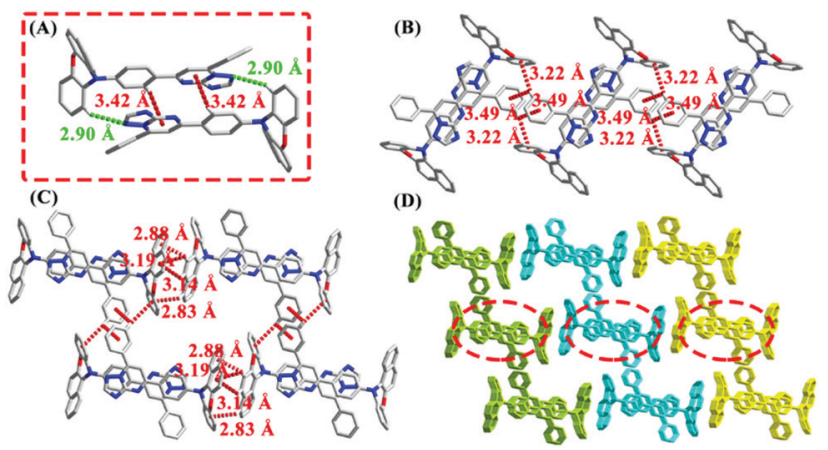

Fig. 3 The packing mode of the 7PTP-BPXZ crystal and intermolecular interactions in the dimers $(\mathrm{C}-\mathrm{H} \cdots \pi$ interactions: red, $\mathrm{C}-\mathrm{H} \cdots \mathrm{N}$ hydrogen bonds: green). Hydrogen atoms except $\mathrm{H} 3, \mathrm{H} 4, \mathrm{H} 10, \mathrm{H} 15, \mathrm{H} 21$ and $\mathrm{H} 30$ were omitted for clarity. 


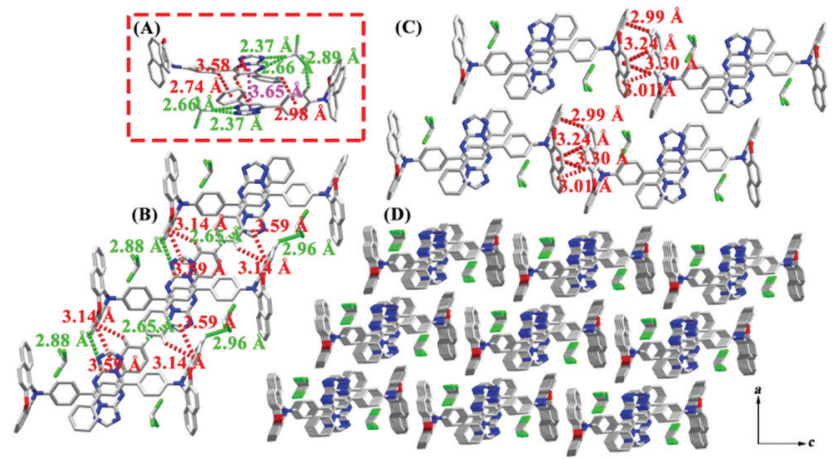

Fig. 4 The packing mode of 5PTP-BPXZ crystal and intermolecular interactions in dimers $(\mathrm{C}-\mathrm{H} \cdots \pi$ interactions: red, $\mathrm{C}-\mathrm{H} \cdots \mathrm{N}$ hydrogen bonds: green, $\pi \cdots \pi$ interactions: purple). Hydrogen atoms except $\mathrm{H} 1 \mathrm{~S}$, $\mathrm{H} 5, \mathrm{H} 6, \mathrm{H} 7, \mathrm{H} 13, \mathrm{H} 14, \mathrm{H} 19, \mathrm{H} 22, \mathrm{H} 24, \mathrm{H} 30, \mathrm{H} 32, \mathrm{H} 33$ and $\mathrm{H} 34$ were omitted for clarity.

Meanwhile, three kinds of strong $\mathrm{C}-\mathrm{H} \cdots \pi$ interactions and a weak $\pi \cdots \pi$ interaction existed in the dimers (Fig. 4A). Then, they were linked by $\mathrm{C} 13-\mathrm{H} 13 \cdots \mathrm{N} 4(d=2.88 \AA), \mathrm{C} 14-\mathrm{H} 14 \cdots \mathrm{Cl} 3(d=2.96 \AA)$, C32-H32 $\cdots \mathrm{N} 2(d=2.65 \AA), \mathrm{C} 33-\mathrm{H} 33 \cdots \pi$ (ring6, C11-C12-C13C14-C15-C16, $d=3.14 \AA$ ) and C34-H34 $\cdots \pi$ (ring6, $d=3.59 \AA$ ), and facilitated the self-assembling into a 1D chain (Fig. 4B and Table S2, $\mathrm{ESI} \dagger$ ). Furthermore, 2D layers were arranged by four types of $\mathrm{C}-\mathrm{H} \cdots \pi$ interactions (Fig. $4 \mathrm{C}$ and $\mathrm{D}$ ), such as $\mathrm{C} 5-\mathrm{H} 5 \cdots \pi$ (ring4, C1-C2-C3-C4-C5-C6, $d=3.24 \AA$ ), C6-H6 $\cdots \pi($ ring4,$d=3.30 \AA$ ), $\mathrm{C} 6-\mathrm{H} 6 \cdots \pi$ (ring5, C3-C4-C7-C8-C9-C10, $d=3.01 \AA$ ) and C7-H7 $\cdots \pi$ (ring6, $d=2.99 \AA$ ). The multiple weak interactions in the crystal lattice restricted intramolecular motions and allowed the excited-state energy to dissipate via radiative relaxation, thus resulting in bright fluorescence.

Crystal 5,7PTP-BPXZ was a monoclinic system with the space group $P 21 / n$. As illustrated in Fig. 5A, the molecules were

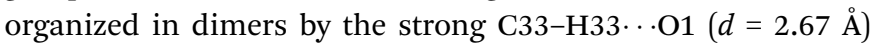
hydrogen bond, C27-H27 $\cdots \pi$ (ring1, C11-C12-C13-C14-C15$\mathrm{C} 16, d=2.97 \AA$ ), C32-H32 $\cdots \pi$ (ring2, C1-C2-C7-C8-C9-C10, $d=3.42 \AA$ ), C33-H33 $\cdots \pi$ (ring2, $d=3.59 \AA$ ) and $\pi \cdots \pi$ (ring3, C17-C18-C19-C20-C21-C22, and ring4, C23-C24-C25-N4C26-N2, $d=3.61 \AA$ ) interactions. Moreover, a 3D staggered pattern was constructed via the adjacent dimers with abundant $\mathrm{C} 3-\mathrm{H} 3 \cdots \mathrm{O} 2(d=2.61 \AA), \mathrm{C} 49-\mathrm{H} 49 \cdots \pi(\operatorname{ring} 4, d=3.40 \AA)$ and $\pi \cdots \pi$ interactions (ring4 and C43-C44-C45-C46-C47-C49, $d=3.59 \AA$ ). These weak interactions could rigidify the molecular structure and largely alleviate the nonradiative decay pathway, which was conducive to enhancing the emission efficiency in condensed states.

\section{High contrast mechanochromism and acidochromism}

As defined in Fig. 6, 7PTP-BPXZ and 5PTP-BPXZ showed distinct tricolor mechanochromic behavior in response to external stimuli. The pristine powder of 7PTP-BPXZ gave sky-blue emission under a $365 \mathrm{~nm}$ UV lamp and the maximum emission wavelength $\left(\lambda_{\mathrm{em}}\right)$ was $472 \mathrm{~nm}$ (Fig. S4, ESI $\dagger$ ). After grinding with a pestle, the powder exhibited deep yellow luminescence and underwent a notable bathochromic shift $\left(\lambda_{\mathrm{em}}=586 \mathrm{~nm}, \Delta \lambda_{\mathrm{em}}=114 \mathrm{~nm}\right)$,

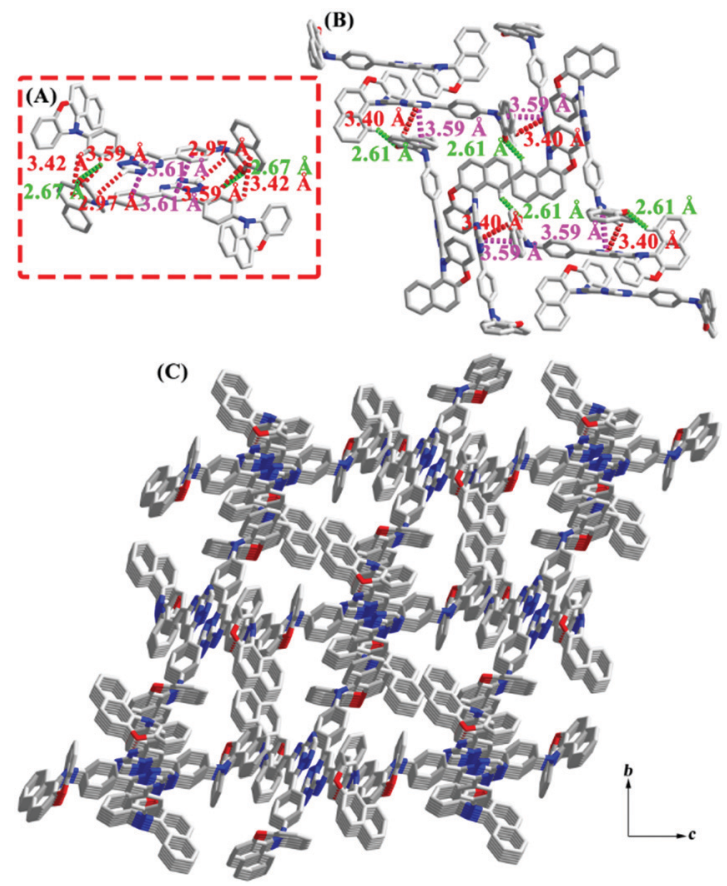

Fig. 5 The packing mode of 5,7PTP-BPXZ crystal and intermolecular interactions in dimers $(\mathrm{C}-\mathrm{H} \cdots \pi$ interactions: red, $\mathrm{C}-\mathrm{H} \cdots \mathrm{N}$ hydrogen bonds: green, $\pi \cdots \pi$ interactions: purple). Hydrogen atoms except $\mathrm{H} 3$, $\mathrm{H} 27, \mathrm{H} 32, \mathrm{H} 33$ and $\mathrm{H} 49$ were omitted for clarity.

(A)

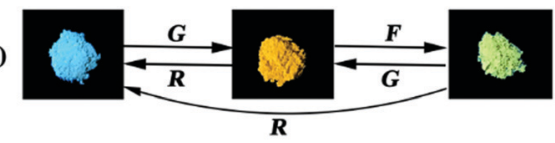

(B)

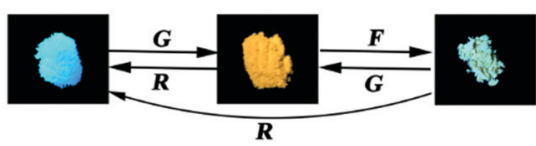

(C)

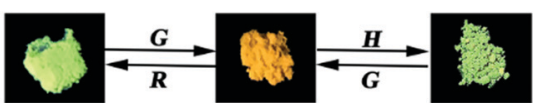

$\boldsymbol{G}$ : Grinding with a mortar and a pestle.

F: Fuming with $\mathrm{CH}_{2} \mathrm{Cl}_{2}$ vapor

H: Heating at $90{ }^{\circ} \mathrm{C}$.

$\boldsymbol{R}$ : Recrystallization from ethyl acetate and petroleum ether.

Fig. 6 Schematic diagrams of the mechanochromic behaviors of 7PTPBPXZ (A), 5PTP-BPXZ (B) and 5,7PTP-BPXZ (C). Fluorescence images were taken under a $365 \mathrm{~nm}$ UV lamp.

which revealed its high contrast mechanochromic characteristic. More impressively, the ground powder became green-emitting $\left(\lambda_{\mathrm{em}}=492 \mathrm{~nm}\right)$ when fuming with dichloromethane $\left(\mathrm{CH}_{2} \mathrm{Cl}_{2}\right)$ vapor (Fig. 6A). The green state could revert back to the ground state by grinding. Besides, the ground or fumed samples of 7PTP-BPXZ both could return to the pristine state by recrystallizing from an ethyl acetate (EA) and petroleum ether (PE) mixture. Similarly, 5PTP-BPXZ showed blue, orange and bluish green switching mechanochromic behavior (Fig. 6B). The maximum emission wavelengths of the pristine, ground and fumed samples were $462 \mathrm{~nm}, 599 \mathrm{~nm}$, and $488 \mathrm{~nm}$ respectively (Fig. S4, ESI $\dagger$ ), displaying 


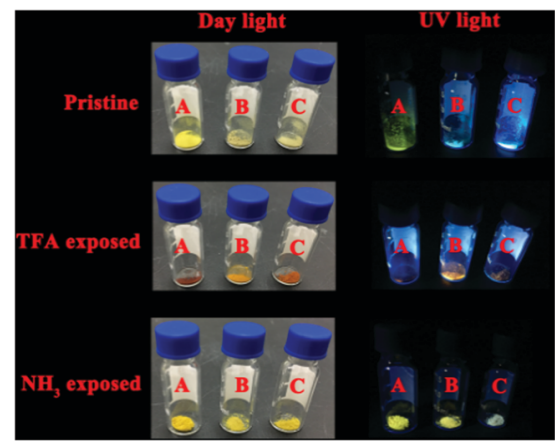

Fig. 7 On-off fluorescence switching of the luminogens by TFA and $\mathrm{NH}_{3}$ exposure under daylight and $365 \mathrm{~nm}$ light. A: 5,7PTP-BPXZ. B: 7PTP-BPXZ. C: 5PTP-BPXZ

better mechanochromic contrast $\left(\Delta \lambda_{\mathrm{em}}=137 \mathrm{~nm}\right)$ than 7PTP-BPXZ. The blue emission of pristine 5PTP-BPXZ powder turned to orange by grinding. When fuming the orange sample with $\mathrm{CH}_{2} \mathrm{Cl}_{2}$ vapor, it became bluish green emission. Conversely, the bluish green state could change to the orange state by grinding. Moreover, the ground and fumed samples of 5PTP-BPXZ could also recover to the pristine state by recrystallizing from an EA and PE mixture. 5,7PTP-BPXZ showed reversible bicolor MCL properties. As shown in Fig. 6C, its pristine powder exhibited green emission $\left(\lambda_{\mathrm{em}}=536 \mathrm{~nm}\right)$. However, the sample showed pronounced red-shifted fluorescence $\left(\lambda_{\mathrm{em}}=\right.$ $602 \mathrm{~nm}$ ) after grinding and could also revert back to the pristine green state by recrystallizing or heating.

Besides, all luminogens displayed reversible acidochromic properties in their solid states (Fig. 7). Taking 7PTP-BPXZ as an example, upon exposure to the vapor of trifluoroacetic acid (TFA) for ten seconds, the maximum emission wavelength of the pristine sample was changed from $472 \mathrm{~nm}$ to $604 \mathrm{~nm}$ with a significant red-shift of $132 \mathrm{~nm}$ (Fig. S4, ESI†). The color transition after fuming with TFA could be easily distinguished by the naked eye in daylight (Fig. 7B), representing high-contrast acidochromism possibly originated from the amine protonation of the PTP moiety.

Powder X-ray diffraction (PXRD) and differential scanning calorimetry (DSC) measurements were performed to acquire deeper insight into their underlying mechanisms of mechanochromism and acidochromism. For instance, the pristine sample of 7PTP-BPXZ exhibited sharp and intense diffraction peaks at around $9^{\circ}$, demonstrating its good crystallinity (Fig. 8). However, the peaks disappeared after grinding, indicating that
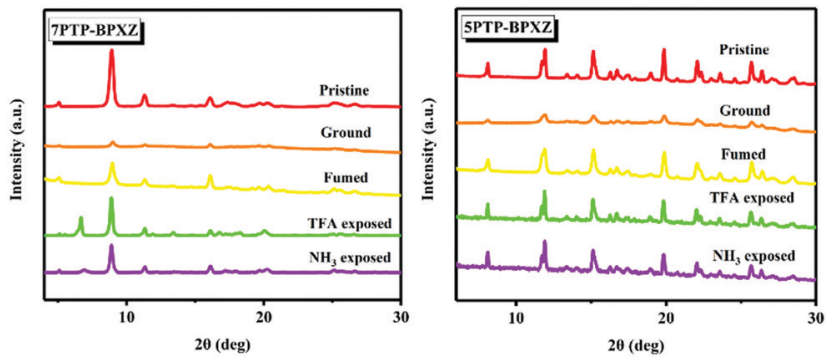

Fig. 8 PXRD curves of luminogens in different states. the lattice of 7PTP-BPXZ was destroyed by mechanical forces. ${ }^{10}$ When fuming by $\mathrm{CH}_{2} \mathrm{Cl}_{2}$ vapor, the original sharp signals were restored, manifesting the recovery of the lattice. Furthermore, the diffraction curves of TFA and $\mathrm{NH}_{3}$ vapor treated samples were not obviously altered compared with those of the pristine samples, indicative of their consistent crystalline patterns. DSC curves illustrated that the melting point of 7PTP-BPXZ was $226{ }^{\circ} \mathrm{C}$ (Fig. S5, ESI $\dagger$ ).

Additionally, the ground sample showed an exothermic recrystallization point at $155{ }^{\circ} \mathrm{C}$. In contrast, the pristine samples showed no such exothermic signal. This revealed that the thermodynamically-stable states were converted into the metastable states by grinding and thus the pristine crystal phase was destroyed.

\section{Conclusions}

In summary, we have developed the first example of singlecomponent metal-free small molecules with both multicolor and high contrast $\left(\Delta \lambda_{\mathrm{em}, \max }>100 \mathrm{~nm}\right)$ mechanochromism and acidochromism. The folded conformation of benzo[a]phenoxazine substitution and the introduction of multiple nitrogen atoms in the triazolopyrimidine moiety have a synergistic effect on these excellent MCL features. As demonstrated by their crystal packing arrangements, the AIE behaviors of the three luminogens can be ascribed to the multiple intermolecular non-covalent interactions which restrict the molecular motion. We believe that the rational molecular design strategy towards multicolor controllable mechanochromic and acidochromic luminogens with distinct emission color contrast will provide a valuable insight into the development of organic intelligent luminescent materials.

\section{Experimental}

\section{Materials and apparatus}

Toluene was dried by sodium-potassium alloy. The other reagents and solvents were used as received from commercial sources without further purification. ${ }^{1} \mathrm{H}$ NMR and ${ }^{13} \mathrm{C}$ NMR spectra were recorded by Bruker Advanced II ( $400 \mathrm{MHz})$ spectrometers and MERCURYVX300 using $\mathrm{CDCl}_{3}$ or $\mathrm{CD}_{3} \mathrm{OD}$ as solvents and tetramethylsilane as the internal standards. High resolution mass spectra were measured on a LCQ-Orbitrap Elite (ThermoFisher Scientific, Waltham, MA, USA) mass spectrometer. UV-vis absorption spectra were collected on a Shimadzu UV-2700 spectrophotometer. Photoluminescence (PL) spectra were recorded on a Hitachi F-4600 fluorescence spectrophotometer. Differential scanning calorimetry (DSC) was performed on a NETZSCH DSC $200 \mathrm{PC}$ unit at a heating rate of $10{ }^{\circ} \mathrm{C} \mathrm{min}{ }^{-1}$ under argon. Powder X-ray diffraction (PXRD) patterns were recorded on a Rigaku (Ultima IV) diffractometer. Single crystal X-ray diffraction (SXRD) data were measured on a Bruker Smart APEX CCD-based diffractometer with graphite-monochromatic Mo K $\alpha$ radiation $(\lambda=0.71073 \AA$ Å). Cyclic voltammetry (CV) was carried out in nitrogen-purged THF (oxidation scan) at room temperature with a CHI voltammetric 
analyser. Tetrabutylammonium hexafluorophosphate (TBAPF6) $(0.1 \mathrm{M})$ was used as the supporting electrolyte. The conventional three-electrode configuration consists of a platinum working electrode, a platinum wire auxiliary electrode, and an $\mathrm{Ag}$ wire pseudo-reference electrode with ferrocenium-ferrocene $\left(\mathrm{Fc}^{+} / \mathrm{Fc}\right)$ as the internal standard. Cyclic voltammograms were obtained at a scan rate of $100 \mathrm{mV} \mathrm{s}^{-1}$. Formal potentials are calculated as the average of cyclic voltammetric anodic and cathodic peaks. CCDC numbers, 1994907 for 7PTP-BPXZ, 1994908 for 5PTP-BPXZ, 1994909 for 5,7PTP-BPXZ.

\section{Synthesis}

Synthesis of $12 H$-benzo[a]phenoxazine (BPXZ). A mixture of $0.31 \mathrm{~g}$ (1.0 mmol) 2-((1-bromonaphthalen-2-yl)oxy)aniline, $0.12 \mathrm{~g}$ (1.2 mmol) sodium tert-butoxide, $0.02 \mathrm{~g}(0.1 \mathrm{mmol})$ palladium(II) acetate and $0.06 \mathrm{~g}(0.2 \mathrm{mmol})$ tri-tert-butylphosphine tetrafluoroborate was dissolved in dry toluene $(20 \mathrm{~mL})$ under an argon atmosphere. After stirring at $110{ }^{\circ} \mathrm{C}$ overnight, the suspension was mixed thoroughly with dichloromethane and washed with water three times. After drying with anhydrous $\mathrm{Na}_{2} \mathrm{SO}_{4}$, the organic phase was concentrated and the crude product was purified by column chromatography on silica gel with petroleum ether/dichloromethane as the eluent. The product was obtained as a greenish yellow solid. Yield: $68 \%$. ${ }^{1} \mathrm{H}$ NMR $(400 \mathrm{MHz}$, $\left.\mathrm{CD}_{3} \mathrm{OD}\right) \delta$ (ppm): 7.83-7.81 (m, 1H), 7.67-7.65 (m, 1H), 7.40$7.31(\mathrm{~m}, 1 \mathrm{H}), 7.30-7.29(\mathrm{~m}, 1 \mathrm{H}), 7.18(\mathrm{~d}, J=8.7 \mathrm{~Hz}, 1 \mathrm{H}), 6.86(\mathrm{~d}, J=$ $8.7 \mathrm{~Hz}, 1 \mathrm{H}), 6.75-6.71(\mathrm{~m}, 1 \mathrm{H}), 6.68-6.64(\mathrm{~m}, 1 \mathrm{H}), 6.63-6.56$ (m, 2H).

Synthesis of 12-(4-(7-phenyl-[1,2,4]triazolo[1,5-a]pyrimidin5-yl)phenyl)-12H-benzo[a]phenoxazine (7PTP-BPXZ). A mixture of $0.11 \mathrm{~g}$ (0.30 mmol) 5-(4-bromophenyl)-7-phenyl-[1,2,4]triazolo[1,5- $a$ ]pyrimidine, $0.08 \mathrm{~g}(0.33 \mathrm{mmol})$ BPXZ, $0.04 \mathrm{~g}$ (0.40 mmol) sodium tert-butoxide, $0.01 \mathrm{~g}(0.03 \mathrm{mmol})$ palladium(II) acetate and $0.02 \mathrm{~g}(0.06 \mathrm{mmol})$ tri-tert-butylphosphine tetrafluoroborate was dissolved in dry toluene $(5 \mathrm{~mL})$ under an argon atmosphere. After stirring at $110{ }^{\circ} \mathrm{C}$ overnight, the suspension was mixed thoroughly with dichloromethane and washed with water four times. After drying with anhydrous $\mathrm{Na}_{2} \mathrm{SO}_{4}$, the organic phase was concentrated and the crude product was purified by column chromatography on silica gel with petroleum ether/ethyl acetate as the eluent. The product was obtained as a yellow solid, which was further crystallized from ethyl acetate. Yield: $81 \%$. ${ }^{1} \mathrm{H}$ NMR (400 $\left.\mathrm{MHz}, \mathrm{CDCl}_{3}\right) \delta(\mathrm{ppm}): 8.47(\mathrm{~s}, 1 \mathrm{H})$, 8.08-8.03 (m, 4H), 7.92-7.87 (m, 2H), 7.78 (d, $J=8.8$ Hz, 1H), 7.70$7.63(\mathrm{~m}, 1 \mathrm{H}), 7.61-7.57$ (m, 3H), 7.51-7.39 (m, 4H), 7.26-7.19 (m, $3 \mathrm{H}), 7.15$ (d, $J=9.0 \mathrm{~Hz}, 2 \mathrm{H}) .{ }^{13} \mathrm{C}$ NMR $\left(100 \mathrm{MHz} \mathrm{CDCl}_{3}\right) \delta(\mathrm{ppm})$ : 161.0, 156.0, 153.8, 151.6, 151.5, 147.7, 133.8, 131.6, 131.1, 129.5, 129.4, 129.2, 128.9, 128.7, 128.4, 127.4, 127.0, 126.6, 126.2, 125.0, 123.8, 122.9, 118.0, 117.7, 117.5, 106.0. HRMS (ESI): $m / z[\mathrm{M}+\mathrm{H}]^{+}$ calcd for $\mathrm{C}_{33} \mathrm{H}_{22} \mathrm{~N}_{5} \mathrm{O}^{+}$: 504.1819; found: 504.1813 .

Synthesis of 12-(4-(5-phenyl-[1,2,4]triazolo[1,5-a]pyrimidin7-yl)phenyl)-12H-benzo[a]phenoxazine (5PTP-BPXZ). 5PTP-BPXZ was synthesized in the same way as described for the synthesis of 7PTP-BPXZ. Yield: $69 \%$. ${ }^{1} \mathrm{H}$ NMR (400 $\left.\mathrm{MHz}, \mathrm{CDCl}_{3}\right) \delta(\mathrm{ppm})$ : $8.47(\mathrm{~s}, 1 \mathrm{H}), 8.24-8.16(\mathrm{~m}, 2 \mathrm{H}), 8.03-7.94(\mathrm{~m}, 3 \mathrm{H}), 7.93-7.88$ (m, 1H), $7.81(\mathrm{dd}, J=9.0,0.7 \mathrm{~Hz}, 1 \mathrm{H}), 7.70-7.63(\mathrm{~m}, 1 \mathrm{H}), 7.56-7.41$ (m, 7H), 7.30-7.26 (m, 2H), 7.25-7.23 (m, 1H), 7.21-7.14 (m, 2H). ${ }^{13} \mathrm{C} \mathrm{NMR}\left(100 \mathrm{MHz}, \mathrm{CDCl}_{3}\right) \delta$ (ppm): 161.4, 156.1, 154.1, 151.9, 151.6, $147.7,136.6,133.4,131.2$, 131.0, 130.3, 129.5, 129.0, 128.5, 127.7, 127.6, 127.2, 127.0, 126.9, 126.0, 125.1, 123.8, 122.8, 122.6, 118.0, 117.6, 116.8, 105.4. HRMS (ESI): $m / z[\mathrm{M}+\mathrm{H}]^{+}$calcd for $\mathrm{C}_{33} \mathrm{H}_{22} \mathrm{~N}_{5} \mathrm{O}^{+}$: 504.1819; found: 504.1813 .

Synthesis of $12,12^{\prime}-([1,2,4]$ triazolo[1,5-a]pyrimidine-5,7-diylbis(4,1-phenylene))bis(12H-benzo[a]phenoxazine) (5,7PTP-BPXZ). 5,7PTP-BPXZ was synthesized in the same way as described for the synthesis of 7PTP-BPXZ. Yield: 85\%. ${ }^{1} \mathrm{H}$ NMR $(400 \mathrm{MHz}$, $\left.\mathrm{CDCl}_{3}\right) \delta(\mathrm{ppm}): 8.39$ (s, 1H), 7.99 (d, $\left.J=9.0 \mathrm{~Hz}, 2 \mathrm{H}\right), 7.95-7.85$ $(\mathrm{m}, 6 \mathrm{H}), 7.78(\mathrm{t}, J=9.1 \mathrm{~Hz}, 2 \mathrm{H}), 7.64-7.62(\mathrm{~m}, 2 \mathrm{H}), 7.52-7.36$ $(\mathrm{m}, 7 \mathrm{H}), 7.27(\mathrm{~s}, 1 \mathrm{H}), 7.25-7.19(\mathrm{~m}, 5 \mathrm{H}), 7.16-7.11(\mathrm{~m}, 4 \mathrm{H})$. ${ }^{13} \mathrm{C}$ NMR (100 MHz, $\left.\mathrm{CDCl}_{3}\right) \delta$ (ppm): 160.7, 155.8, 154.1, 153.9, 153.7, 151.8, 151.5, 151.4, 147.3, 133.9, 131.1, 131.0, 130.2, 129.9, 129.5, 129.3, 128.6, 128.4, 128.3, 127.6, 127.4, 127.1, 126.9, 126.8, $126.5,126.4,126.2,126.0,125.8,124.9,123.8,122.9,122.8,118.0$, 117.9, 117.8, 117.6, 117.4, 116.8, 104.8. HRMS (ESI): $m / z[\mathrm{M}+\mathrm{H}]^{+}$ calcd for $\mathrm{C}_{49} \mathrm{H}_{31} \mathrm{~N}_{6} \mathrm{O}_{2}^{+}$: 735.2503; found: 735.2505.

\section{Conflicts of interest}

There are no conflicts to declare.

\section{Acknowledgements}

This work was supported by grants from the National Natural Science Foundation of China (No. 21721005, 91833304, 21805195 and 51903159), the Shenzhen Science and Technology Program (KQTD20170330110107046) and the Shenzhen Technology and Innovation Commission (JCYJ20180507182244027). The numerical calculations in this paper were carried out on the supercomputing system in the Supercomputing Center of Wuhan University.

\section{Notes and references}

1 (a) J. Zhang, A. Li, H. Zou, J. Peng, J. Guo, W. Wu, H. Zhang, J. Zhang, X. Gu, W. Xu, S. Xu, S. H. Liu, A. Qin, J. W. Y. Lam and B. Z. Tang, A simple donor-acceptor AIEgen with multistimuli responsive behavior, Mater. Horiz., 2020, 7, 135-142; (b) W. Zhao, Z. He, Q. Peng, J. W. Y. Lam, H. Ma, Z. Qiu, Y. Chen, Z. Zhao, Z. Shuai, Y. Dong and B. Z. Tang, Highly sensitive switching of solid-state luminescence by controlling intersystem crossing, Nat. Commun., 2018, 9, 3044-3051; (c) K. Zheng, H. Yang, F. Ni, Z. Chen, S. Gong, Z. Lu and C. Yang, Multifunctional thermally activated delayed fluorescence emitters and insight into multicolor-mechanochromism promoted by weak intra- and intermolecular interactions, $A d v$. Opt. Mater., 2019, 7, 1900727-1900735; (d) W. Yang, Y. Yang, L. Zhan, K. Zheng, Z. Chen, X. Zeng, S. Gong and C. Yang, Polymorphism-dependent thermally activated delayed fluorescence materials with diverse three dimensional supramolecular frameworks, Chem. Eng. J., 2020, 390, 124626-124634; (e) B. Roy, M. C. Reddy and P. Hazra, Developing the 
structure-property relationship to design solid state multistimuli responsive materials and their potential applications in different fields, Chem. Sci., 2018, 9, 3592-3606.

2 (a) G. Huang, Q. Xia, W. Huang, J. Tian, Z. He, B. S. Li and B. Z. Tang, Multiple anti-counterfeiting guarantees from a simple tetraphenylethylene derivative high-contrasted and multi-state mechanochromism and photochromism, Angew. Chem., Int. Ed., 2019, 58, 17814-17819; (b) K. Zheng, F. Ni, Z. Chen, C. Zhong and C. Yang, Polymorph-dependent thermally activated delayed fluorescence emitters: Understanding TADF from a perspective of aggregation state, Angew. Chem., Int. Ed., 2019, 58, 1-6; (c) X. Zheng, W. Zhu, C. Zhang, Y. Zhang, C. Zhong, H. Li, G. Xie, X. Wang and C. Yang, Self-assembly of a highly emissive pure organic imine-based stack for electroluminescence and cell imaging, J. Am. Chem. Soc., 2019, 141, 4704-4710; (d) Z. Yang, Z. Chi, Z. Mao, Y. Zhang, S. Liu, M. P. Aldred and Z. Chi, Recent advances in mechano-responsive luminescence of tetraphenylethylene derivatives with aggregation-induced emission properties, Mater. Chem. Front., 2018, 2, 861-890.

3 J. Zhao, Z. Chi, Y. Zhang, Z. Mao, Z. Yang, E. Ubba and Z. Chi, Recent progress in the mechanofluorochromism of cyanoethylene derivatives with aggregation-induced emission, J. Mater. Chem. C, 2018, 6, 6327-6353.

4 J. Luo, Z. Xie, J. W. Y. Lam, L. Cheng, H. Chen, C. Qiu, H. S. Kwok, X. Zhan, Y. Liu, D. Zhu and B. Z. Tang, Aggregationinduced emission of 1-methyl-1,2,3,4,5-pentaphenylsilole, Chem. Commun., 2001, 1740-1741.

5 (a) D. T. Hogan, B. S. Gelfand, D. M. Spasyuk and T. C. Sutherland, Subtle substitution controls the rainbow chromatic behaviour of multi-stimuli responsive core-expanded pyrenes, Mater. Chem. Front., 2020, 4, 268-276; (b) J. Zhu, C. Li, P. Chen, Z. Ma, B. Zou, L. Niu, G. Cui and Q. Yang, A polymorphic fluorescent material with strong solid state emission and multi-stimuli-responsive properties, Mater. Chem. Front., 2020, 4, 176-181; (c) B. Huang, X. Gu, Y. Feng, Y. Zhang, D. Jiang, W. C. Chen, G. Dai, Y. Ji, Q. Zhao and C. S. Lee, Solid-state fluorophore based on $\pi$-extended heteroaromatic acceptor: Polymorphism, mechanochromic luminescence, and electroluminescence, Cryst. Growth Des., 2020, 20, 2454-2461; (d) H. Tian, P. Wang, J. Liu, Y. Duan and Y. Q. Dong, Construction of a tetraphenylethene derivative exhibiting high contrast and multicolored emission switching, J. Mater. Chem. C, 2017, 5, 12785-12791.

6 G. Huang, Y. Jiang, S. Yang, B. S. Li and B. Z. Tang, Multistimuli response and polymorphism of a novel tetraphenylethylene derivative, Adv. Funct. Mater., 2019, 29, 1900516-1900524.

7 M. Okazaki, Y. Takeda, P. Data, P. Pander, H. Higginbotham, A. P. Monkman and S. Minakata, Thermally activated delayed fluorescent phenothiazine-dibenzo[ $[a, j]$ phenazine-phenothiazine triads exhibiting tricolor-changing mechanochromic luminescence, Chem. Sci., 2017, 8, 2677-2686.

8 X. Zeng, T. Zhou, J. Liu, K. Wu, S. Li, X. Xiao, Y. Zhang, S. Gong, G. Xie and C. Yang, Incorporating thermally activated delayed fluorescence into mechanochromic luminescent emitters: highperformance solution-processed yellow organic light emitting diodes, Adv. Opt. Mater., 2018, 6, 1801071-1801079.

9 M. D. Yang, D. L. Xu, W. G. Xi, L. K. Wang, J. Zheng, J. Huang, J. Y. Zhang, H. P. Zhou, J. Y. Wu and Y. P. Tian, Aggregation-induced fluorescence behavior of triphenylaminebased schiff bases: the combined effect of multiple forces, J. Org. Chem., 2013, 78, 10344-10359.

10 (a) W. Yang, C. Liu, S. Lu, J. Du, Q. Gao, R. Zhang, Y. Liu and C. Yang, AIE-active smart cyanostyrene luminogens: polymorphism-dependent multicolor mechanochromism, J. Mater. Chem. C, 2018, 6, 290-298; (b) Y. Li, Z. Ma, A. Li, W. Xu, Y. Wang, H. Jiang, K. Wang, Y. Zhao and X. Jia, A single crystal with multiple functions of optical waveguide, aggregation-induced emission, and mechanochromism, ACS Appl. Mater. Interfaces, 2017, 9, 8910-8918; (c) J. Mei, N. L. C. Leung, R. T. K. Kwok, J. W. Y. Lam and B. Z. Tang, Aggregationinduced emission: together we shine, united we soar, Chem. Rev., 2015, 115, 11718-11940; (d) L. Pan, Y. Cai, H. Wu, F. Zhou, A. Qin, Z. Wang and B. Z. Tang, Tetraphenylpyrazine-based luminogens with full-colour emission, Mater. Chem. Front., 2018, 2, 1310-1316. 